\title{
Statistical modelling of extreme ocean climate with incorporation of storm clustering
}

\author{
$\underline{\text { Wenping Jiang }}{ }^{\text {a }}$, Gareth Davies ${ }^{a}$, David P. Callaghan ${ }^{\mathrm{b}}$, Tom Baldock ${ }^{\mathrm{b}}$ and Scott Nichol ${ }^{\mathrm{a}}$ \\ ${ }^{a}$ Geoscience Australia, GPO Box 378, Canberra, ACT 2601, Australia \\ ${ }^{b}$ The University of Queensland, Brisbane, QLD 4072, Australia \\ Email: wenping.jiang@ga.gov.au
}

\begin{abstract}
Knowledge of the extreme ocean climate is essential for the accurate assessment of coastal hazards to facilitate risk informed decision making in coastal planning and management. Clustered storm events, where two or more storms occur within a relatively short space of time, may induce disproportionately large coastal erosion compared to non-clustered storm events. Therefore this study aims to develop a statistical approach to modelling the frequency and intensity of storm events on the eastern and southern coast of Australia, with a focus on examining storm clustering. This paper presents the preliminary analysis of the recently developed methods and results when they are applied to a study site on the central coast of New South Wales, Australia. This study is a key component of the Bushfire and Natural Hazards CRC Project "Resilience to clustered disaster events on the coast - storm surge" that aims to develop a new method to quantify the impact of coincident and clustered disaster events on the coast.
\end{abstract}

Extreme storm events at a given site can be described using multivariate summary statistics, including the events' maximum significant wave height $\left(\mathrm{H}_{\text {sig }}\right)$, median wave period, median wave direction, duration, peak storm surge, and time of occurrence. This requires a definition of individual storm events, so the current methodology firstly involves the extraction of storm events from a 30-year timeseries of observations. Events are initially defined using a peaks-over-threshold approach based on the significant wave height with the $95 \%$ exceedance quantile $(2.93 \mathrm{~m})$ adopted as the threshold. Subsequently, these events are manually checked against sea-level pressure data to examine if closely spaced events are generated by the same meteorological system, and if so the events are combined. This means that the final event set is more likely to consist of meteorologically independent storm events.

Various statistical techniques are applied to model the magnitude and frequency of the extracted storm events. A number of variations on the non-homogenous Poisson process model are developed to estimate the event occurrence rate, duration and spacing. The models account for the sub-annual variations in the occurrence rate, temporal dependency between successive events, and the finite duration of events. The results indicate that in the current dataset, closely spaced events are more temporally spread out than would be expected if the event timings are independent, which we term anti-clustering. A particular marginal distribution is fitted to each variable, i.e. a Generalised Pareto (GP) distribution for $\mathrm{H}_{\text {sig, }}$, and Pearson type 3 (PE3) distributions for duration and tidal residual. Empirical marginal distributions are employed for wave period and direction. The joint cumulative distribution function of all storm magnitude statistics is modelled by constructing the dependency structure using Copula functions. Two methods are tested: a t-copula and a combination of a Gumbel and Gaussian copulas. Comparison of modelled and observed scatterplots shows similar patterns. Goodness-of-fit tests such as Komologorov-Smirnov (K-S) tests, Chi-square tests and AIC and BIC are used to quantitatively evaluate the fitting qualities and to assess model parsimony, along with graphical visualisations, e.g. QQ plots.

Based on this approach, a long-term synthetic time-series of storm events ( $10^{6}$ years) is generated using the event magnitude and timing simulated with the fitted models. These long-term synthetic events can be used to derive exceedance probabilities and to construct designed storm events to be applied to beach erosion modelling.

Keywords: Storm clustering, non-homogenous Poisson process, marginal distribution, Copulas, synthetic events 


\section{INTRODUCTION}

Coastal communities in Australia are exposed to extreme ocean climate because of the narrow continental shelf and the dynamic nature of the coast, with sandy coasts particularly vulnerable to the impact of storm events (Roberts et al. 1995; Coco et al. 2014; Masselink and van Heteren 2014). Knowledge of extreme ocean climate is essential for the accurate assessment of coastal hazards to facilitate risk informed decision making in coastal planning and management. Extreme event timings and magnitudes appear to be stochastic with events characterised by a range of variables, suggesting a multivariate statistical modelling approach, in which the event variables are treated as random and described in terms of probability distributions (Schölzel and Friederichs 2008). Recent developments of such a framework have been applied to assess beach erosion and inundation (e.g. Hawkes et al. 2002; Callaghan et al. 2008; Karunarathna et al. 2014; Li et al. 2014).

Clustered coastal storm events, where two or more storms occur within a relatively short space of time, may induce disproportionately large coastal erosion compared to non-clustered storms. Therefore some efforts have been devoted to estimation of the event frequency with incorporation of temporal dependency. For example, Luceño et al. (2006) have developed a model that contains terms accounting for temporal dependency to simulate storm clustering in the Western Mediterranean Sea. Their result shows that the new model outperforms the non-homogeneous Poisson process approach which does not account for dependency of successive extreme events.

Multivariate techniques are generally required to model extreme ocean climates, which are characterized by storm surge, wave height, wave period and direction. Copula functions have been extensively used in estimation of the associated joint probability distributions. For example, Corbella and Stretch (2012) used copulas to construct a trivariate model of wave height, storm duration and wave period; De Michele et al. (2007) used copulas to provide a four-dimensional, multivariate frequency analysis of wave height, storm duration, direction, and inter-arrival time. Li et al. (2014) compared four copula construction methods to optimise a dependency structure for modelling of wave climate along the Dutch coast.

This study aims to develop a statistical approach to modelling the frequency and intensity of storm events on the eastern and southern coast of Australia. We focus in particular on accounting for temporal dependencies in storm timings, to quantify and assess the significance of storm clustering. This paper presents the preliminary analysis of the recently developed methods applied to the Old Bar site.

\section{STUDY SITE AND DATA}

Old Bar Beach is one of the erosion hotspots located on the central coast of New South Wales (NSW), Australia (Figure 1). It has been subject to a recession rate of $2 \mathrm{~m} /$ year in recent years (Royal Haskoning 2013). Private and public properties are threatened by severe storms, e.g. three houses at the southern end of the coastal subdivision were demolished in 2008 (Royal Haskoning 2013).

The ocean wave regime on the NSW coast is characterised as moderate to high energy (Short and Trenaman 1992). Wave and tidal data has been collected under the NSW Coastal Data Network Program managed by the Office of Environment and Heritage $(\mathrm{OEH})$. The Crowdy Head waverider buoy is the closet to the study site (Figure 1). Data from the Coffs Harbour and Sydney waverider buoys was used to fill gaps in the Crowdy Head data, with Coffs Harbour being used as the first preference since it is more correlated with conditions at Crowdy Head. The Tomaree gauge data is considered to be the most

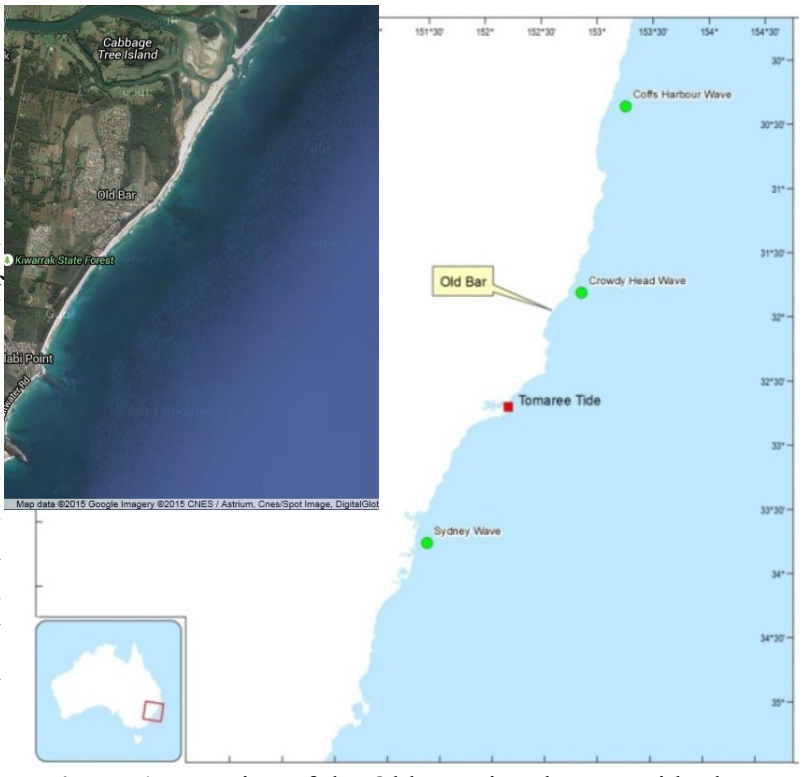

Figure 1. Location of the Old Bar site, the waverider buoys and the tidal gauge that data are sourced from. representative tidal data for the region. TPXO7.2 (http://volkov.oce.orst.edu/tides/global.html) is used to produce astronomical tides for calculating storm surges. Sea-level pressure reanalysis data is provided by the NOAA/OAR/ESRL PSD, Boulder, Colorado, USA (http://www.esrl.noaa.gov/psd/). 


\section{METHOD}

Individual storm events are initially extracted from the timeseries data using a peaks-over-threshold approach based on the significant wave height. The threshold value is set to the $95 \%$ exceedance quantile $(2.93 \mathrm{~m})$, and is comparable to that recommended for the NSW coast $(3 \mathrm{~m}$, Lord and Kulmar 2000). As a first pass, these storm events are extracted from 30-year observational timeseries. Subsequently, these events are manually checked against sea-level pressure maps to examine if closely spaced events (within 10 days) are generated by the same meteorological system, in which case they are combined into a single event. This will reduce the dependence between consecutive events in the data. An example event is given in Figure 2.

Once the individual storm events are determined, each storm event is characterised by a set of summary statistics in terms of maximum significant wave height $\left(\mathrm{H}_{\text {sig }}\right)$, mean wave period (Tp1) and direction, peak tidal residual, and storm duration. Various statistical modelling techniques are applied to simulate the magnitude and frequency of the storm events. Details are given in the following sections.

\subsection{Events Frequency - Non-Homogeneous Poisson Process}

Firstly, time gaps between events and the event occurrence rate over the full data period are assessed. Figure 3 shows an exponential-like distribution of time between events and a notable seasonal pattern in monthly occurrence rates with more storms occurring in the winter months than that in the summer.

Thus, a non-homogeneous Poisson process is adopted to account for the sub-annual variations in the rate of storm occurrence, which leads to the rate parameter $\lambda$ becoming a function of time $\lambda(t)$. Assuming events

Histogram of time between events

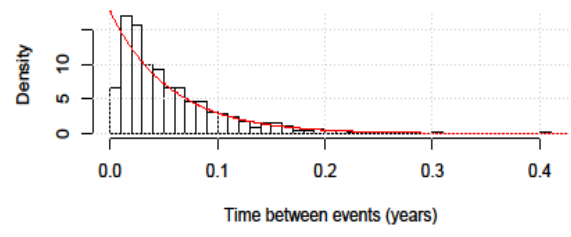

Events occuring in each calendar month

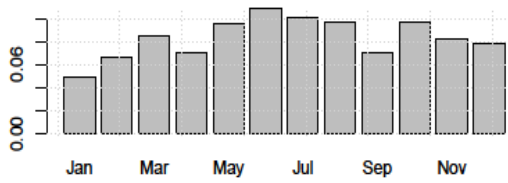

Figure 3. Distribution of the time between

events (Top); and fraction of events occurring in each calendar month (Bottom).
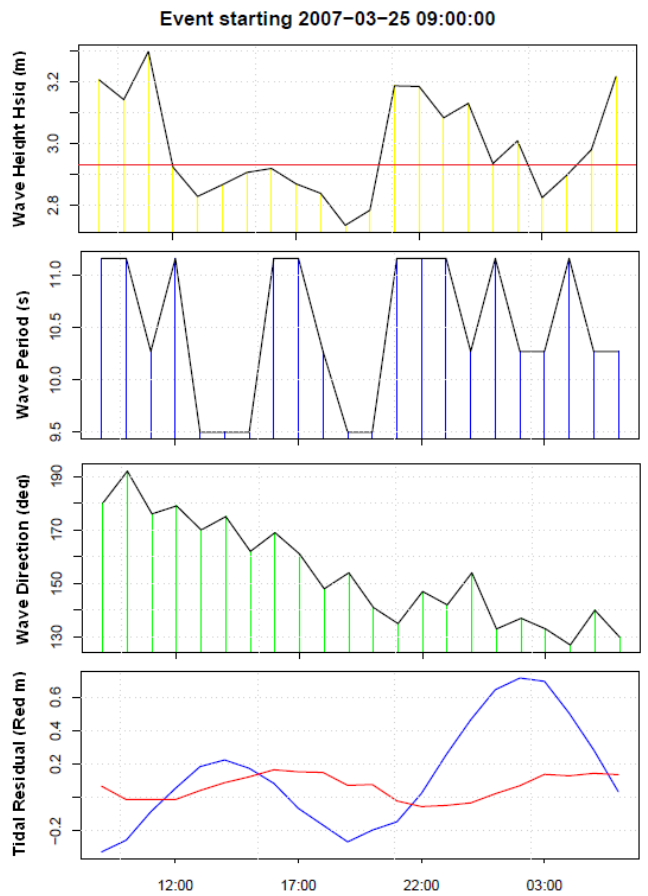

Figure 2. An example of extracted storm events occurring on the $25-26^{\text {th }}$ Mar 2007. occur at times $t_{i} ; \mathrm{i}=1 ; 2 ; 3 ; \ldots$ where $t_{i+1}>t_{i}$, given that the last event occurred at time $t_{i}$, the probability that an event occurs at $t_{i+1}$, is:

$$
F\left(t_{i+1} \mid t_{i}, \lambda(t)\right)=1-\exp \left(-\int_{t_{i}}^{t_{i+1}} \lambda(t) d t\right) \quad \text { Eq. } 1
$$

The function $\lambda(t)$ can then be used to handle temporal variations in the occurrence rate ( $t$ is in the unit of year). In theory, $\lambda(t)$ can be any functional form that represents a temporal pattern in the occurrence rate (given that $\lambda(t)$ is not less than 0 ). For example, Eq. 2 represents a sinusoidal curve with a peak $\lambda$ of $\theta_{1}+\theta_{2}$, a minimum value of $\theta_{1}-\theta_{2}$, and an annual average value of $\theta_{1}$. Here $\theta$ denotes the vector of parameters with appropriate values that can simulate a higher rate in the winter months and a lower rate in summer.

$$
\lambda(t \mid \theta)=\operatorname{Max}\left[\theta_{1}+\theta_{2} \sin \left(2 \pi\left(t-\theta_{3}\right)\right), 0\right] \quad \text { Eq. } 2
$$

To further account for the statistical dependence of temporally close events, an approach proposed by Luceño et al. (2006) is adapted herein, which involves making $\lambda$ a function of both $t$, and the time of the last event $t_{i}$. The sinusoidal example above becomes:

$$
\lambda\left(t \mid \theta, t_{i}\right)=\operatorname{Max}\left[\theta_{1}+\theta_{2} \sin \left(2 \pi\left(t-\theta_{3}\right)\right)+\theta_{4} \exp \left(\theta_{5}\left(t_{i}-t\right)\right), 0\right]
$$

where $t>t_{i}$ and again, $\lambda(t)$ is not less than 0 . This function has an extra exponential term which can increase the rate for times sufficiently close to the last event (assuming $\theta_{4}$ and $\theta_{5}$ are positive). The number $1 / \theta_{5}$ can be interpreted as a timescale over which clustering is important, with the magnitude of $\theta_{4}$ compared with $\theta_{1}$ and $\theta_{2}$ reflecting the importance of clustering. This model can also be applied with $\theta_{4}<0$ which implies anticlustering, or a tendency for less closely spaced events than that would be expected from a standard nonhomogeneous Poisson process. 
Storms have a finite duration, during which time other storms cannot occur (or they will be treated as the same event). To account for events gaps, the above models are adjusted. Suppose the event at $t_{i}$ has a known duration $d_{i}$ and in this time the probability of another event occurring is zero. The model is then:

$$
\begin{aligned}
F\left(t_{i+1} \mid t_{i}, d_{i}, \lambda\left(t \mid \theta, t_{i}+d_{i}\right)\right) & =1-\exp \left(-\int_{t_{i+d_{i}}}^{t_{i+1}} \lambda\left(t \mid \theta, t_{i}+d_{i}\right) d t\right) \text { if } t_{i+1}>t_{i}+d_{i} \\
& =0 \text { otherwise }
\end{aligned}
$$

Eventually, the model is coded in R ( $\mathrm{R}$ Development Core Team 2012). The $\theta$ parameters are estimated using the Maximum Likelihood Estimation (MLE). Diagnostic plots are produced for graphically assessing the model fit to the data. In addition, Komologorov-Smirnov (K-S) tests are used to quantitatively compare the distribution of various quantities in the simulated and actual datasets. Although the associated p-values are over-estimates because the simulated dataset is not independent of the observed data, this approach can nonetheless highlight any strong differences between the modelled and observed datasets. We also use AIC and BIC to assess model parsimony.

\subsection{Events Magnitude - Marginal Distribution and Joint Distribution}

Pairwise scatterplots (Figure 4) and histograms of the key variables highlight the marginal distributions of each variable and their correlations. Note, the variable "season" represents an approximation of seasonal variations as a form of $\cos \left(2 * p i^{*} t\right)$ where $t$ is the event time. $\mathrm{H}_{\text {sig }}$ and duration both show strongly right skewed distributions, while the tidal residual shows a slightly right-skewed distribution. Figure 5 is an L-moment ratio diagram (Hosking and Wallis 1997), which suggests that the Generalised Pareto (GP) or Pearson type 3 (PE3) distributions may be appropriate for $\mathrm{H}_{\text {sig }}$ and duration, while a Gaussian or PE3 distribution may be appropriate for the tidal residual. These distributions are fitted to the data using L-moments, which are often suggested for small-sample studies where they may be more robust than MLE (Hosking and Wallis 1997). For wave

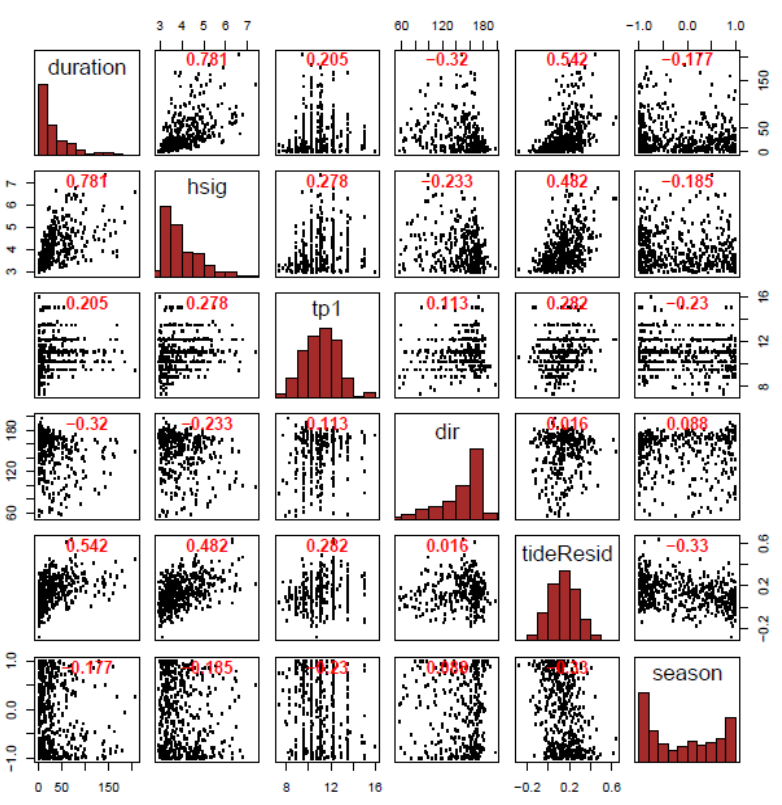

Figure 4. Pairwise scatterplots of event variables including season. The titles give spearman rank correlations.

direction and wave period, empirical distributions are applied. They can be implemented using R's density function, which uses a Gaussian kernel to estimate the density. The bandwidth is adjusted subjectively to make the marginal density sufficiently smooth. K-S tests and graphical visualisations are employed to check the fit of each marginal distribution.

Subsequently, two alternative copula methods are constructed to model the joint distribution of the variables. The first approach is to simulate the dependency structure of the all the variables using tcopula, except for the variable "season" because it is weakly correlated to some of the variables. The t-copula is an elliptical

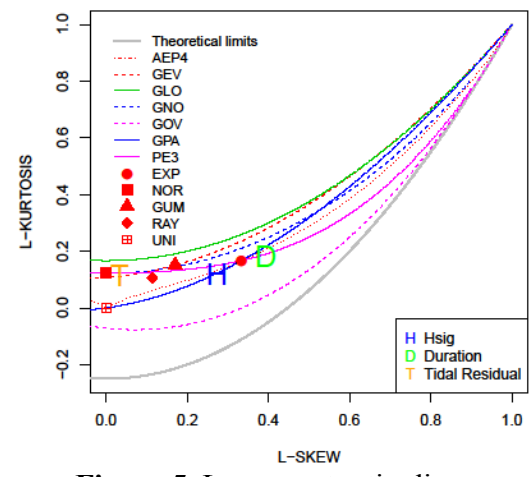
copula, which is similar to a Gaussian copula but has some tail Figure 5. L-moment ratio diagrams. dependence and simply generalises to the multivariate case (Nelsen 1999). The second approach is a combination of two copulas: a Gumbel copula linking $\mathrm{H}_{\text {sig }}$ and duration, and a Gaussian copula linking $\mathrm{H}_{\text {sig }}$ and all other variables. The Gumbel copula, known as a type of Archimedean copula, is an asymmetric copula with greater dependence in the positive tail than in the negative (Gumbel and Mustafi 1967). The Gaussian copula is an elliptical copula with the dependence modelled by means of a symmetric metric that can be easily generalised to higher dimensions using the probability integral transform (Li et al. 2014). The two alternatives are fitted to the data using MLE. Pairwise scatterplots are also produced for visualisation. 


\section{RESULTS}

In terms of simulating the frequency of events, six variations of $\lambda$ on the non-homogenous Poisson process model are tested, including a simple sinusoidal curve (Fit1), a complex sinusoidal curve (Fit2), a triangular equation (Fit3), and these three extended to contain either a positive clustering term (Fit11, Fit21 and Fit31) or negative clustering term (Fit12, Fit22 and Fit32). K-S tests are conducted to compare the fitted and observed event times of year, the time between events, and the number of events per year, and the results are given in Table 1, along with the AIC and BIC values for each fit. Although the associated p-values are over-estimates because the simulated dataset is not independent of the observed data (since the model parameters are estimated from the data), this method can still highlight sufficiently

poorly fitting models. The K-S results suggest that all the models perform well for simulating the event time of year and the number of events per year, whereas only Fit12, Fit22 and Fit32 achieve a reasonable agreement in simulating the time between events, all of which contain a negative clustering term. The AIC/BIC criteria also suggest these three models are the most parsimonious. Graphical examination shows that, except for these three models, others all tend to over-estimate the frequencies of closely spaced events. The diagnostic plots for the model Fit32 are given in Figure 6.

Table 1. K-S test results and associated AIC/BIC criteria values for the models tested.

\begin{tabular}{|c|c|c|c|c|c|}
\hline \multirow[b]{2}{*}{ Models } & \multicolumn{3}{|c|}{ p-value $(\mathrm{K}-\mathrm{S})$} & \multirow[b]{2}{*}{$\mathrm{AIC}$} & \multirow[b]{2}{*}{$\mathrm{BIC}$} \\
\hline & $\begin{array}{l}\text { event } \\
\text { time }\end{array}$ & $\begin{array}{c}\text { time } \\
\text { between } \\
\text { events }\end{array}$ & $\begin{array}{c}\text { number } \\
\text { of } \\
\text { events }\end{array}$ & & \\
\hline Fit1 & 0.77 & $5.5 \mathrm{e}-5$ & 0.78 & -1831 & -1814 \\
\hline Fit11 & 0.58 & $4.3 e-5$ & 0.94 & -1827 & -1802 \\
\hline Fit12 & 0.56 & 0.76 & 0.84 & -1899 & -1874 \\
\hline Fit2 & 0.95 & $5.5 \mathrm{e}-5$ & 0.80 & -1831 & -1806 \\
\hline Fit21 & 0.89 & $9.5 e-5$ & 0.94 & -1827 & -1794 \\
\hline Fit22 & 0.58 & 0.83 & 0.62 & -1903 & -1869 \\
\hline Fit3 & 0.78 & $4.5 \mathrm{e}-5$ & 0.80 & -1832 & -1815 \\
\hline Fit31 & 0.74 & $5.2 \mathrm{e}-5$ & 0.87 & -1828 & -1803 \\
\hline Fit32 & 0.50 & 0.64 & 0.70 & -1901 & -1876 \\
\hline
\end{tabular}

With respect to event magnitude, parametric marginal distributions are fit to $\mathrm{H}_{\text {sig, duration, and }}$ tidal residual, with the distribution selected based on graphical checks. A Generalised Pareto (GP) distribution is selected for $\mathrm{H}_{\text {sig }}$, while Pearson type 3 (PE3) distributions are chosen for duration and tidal residual. Smoothed empirical distributions are used for other variables. Having fitted these marginal distributions, the two copula methods are applied to model the joint distribution of the data and their performances are compared. Comparison of modelled and observed scatterplots shows similar patterns. Efforts have been made to quantify the model performance using Chi-square tests. However, our initial tests suggest the Chisquare statistics depends on the bin selection, which is subjective. In this sense, it is not a reliable goodness-of-fit test. Further assessment of these methods is required.

Eventually, a set of synthetic time-series of storm events $\left(10^{6}\right.$ years $)$ is generated using the event magnitude and timing suggested by the fitted models. QQ plots indicate a reasonable agreement between the simulated and observed storm properties, as shown in Figure 7. These long-term synthetic events can be used to derive exceedance probabilities and to construct designed storm events to be applied to beach erosion modelling. An example 10-year synthetic events series is given in Figure 8, along with the simultaneous observed events.

\section{DISCUSSION}

For a statistical modelling purpose, if storm events are defined purely based on a peaks-over-threshold approach, it is likely that there will be strong dependencies between consecutive events that are challenging to model. By manually checking against sea-level pressure data to identify closely spaced events that are generated by the same meteorological system, the events become more statistically independent. 
The statistical modelling results reflect the site-specific climate characteristics. The models that contain negative clustering terms perform better, indicating a statistically significant anti-clustering pattern in the current dataset. This is in contrast to the finding by Luceño et al. (2006) who have identified temporal dependence in closely spaced extreme storm events in their dataset. They note that temporal dependence can be more pronounced in the protected areas of gulfs where persistent atmospheric situations with low barometric pressures can cause accumulation of water. In our study no such positive temporal dependence is detected, and while this may partly reflect difference in the site characteristics, another key factor is that our methodology involves merging events generated by the same weather system, whereas no such merging was done by Luceño et al. (2006). In addition, the anti-clustering of our data may reflect that the Old Bar site is located on the open coast, subject to ocean waves that travel long distances from the Southern Ocean and Tasman Sea, or from tropical cyclones in the Coral Sea (Short and Trenaman 1992). Successive low-pressure systems from the same origin may diverge in different directions, leading to less closely spaced events received at the Old Bar site. Two low-pressure systems may also merge into a single system before they can generate separate high wave events at the study site, and this should lead to less closely spaced events than expected assuming temporal independence.

The joint cumulative distribution function of all storm magnitude statistics is modelled using Copulas. The t-copula extends the Gaussian copula to allow for some tail dependence and simply generalises to the multivariate case. However the tail dependence has to be similar among all the variables, which may not be realistic in general. The combination of the Gumbel and Gaussian copulas is more flexible. The Gaussian copula has the advantage of simplicity and has been widely applied to extreme value analysis in ocean climate and hydrology (e.g. Hosking and Wallis 1988; Renard and Lang 2007; Schölzel and Friederichs 2008). Li et al. (2014) recommend the Gaussian copula for the Dutch coast after comparing four dependency structures constructed using different copulas. The Gumbel copula is designed to account for upper tail dependence; however, it may be challenging to establish tail dependence in datasets with only a few hundred events (Serinaldi et al. 2015). A limitation of the combined Gumbel and Gaussian copulas approach is that the correlations between duration and variables other than $\mathrm{H}_{\text {sig }}$ can only be controlled indirectly via their dependence on $\mathrm{H}_{\text {sig }}$, hence may be less well modelled than using the t-copula. Other copulas may also perform well depending on the dataset and are worth investigating.
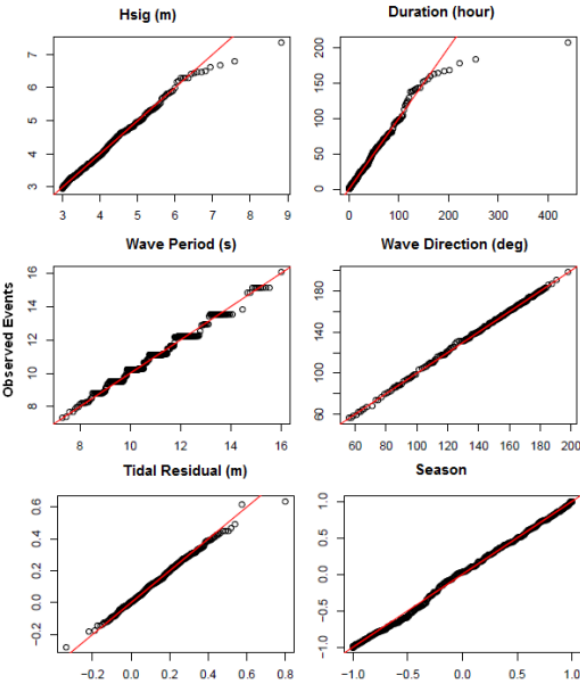

Figure 7. QQ plots of simulated and observed storm events properties.
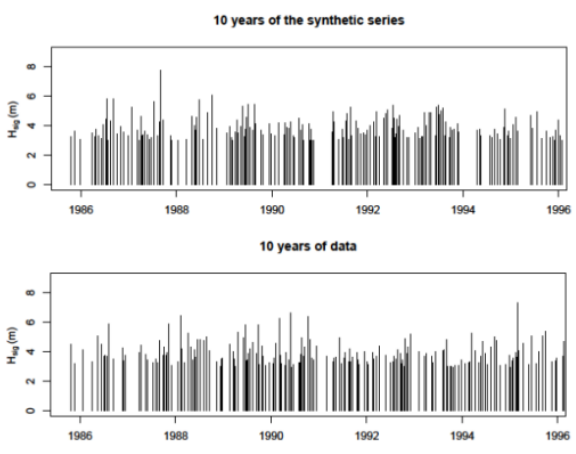

Figure 8. Demonstration of synthetic events and observed events.

The model has the potential to include non-stationary processes such as ENSO, Southern Annular Mode, and Pacific Decadal Oscillation. On-going work is undertaken to identify variation patterns in the data.

\section{CONCLUSIONS}

Firstly, a set of storm events is identified by adopting the peaks-over-threshold approach and manually checking against sea-level pressure data to combine closely spaced events that are generated by the same meteorological system. A number of variations on the non-homogenous Poisson process model are developed to estimate the event occurrence rate, duration and spacing. The models account for the sub-annual variations in the occurrence rate, temporal dependency between successive events, and the finite duration of events. The results show that, for the current dataset, the models that contain terms accounting for an anticlustering type temporal dependency perform better than the non-homogeneous Poisson process approach without dependency of successive extreme events, or with a clustering type dependency. The anti-clustering term for the closely spaced events operates over a relatively short timescale (with an e-folding time of 2.2 days in the current dataset).

A particular marginal distribution is fitted to each storm summary statistic, i.e. a Generalised Pareto (GP) distribution for $\mathrm{H}_{\text {sig }}$, Pearson type 3 (PE3) distributions for duration and tidal residual. Empirical distributions 
are applied to wave period and direction. The joint cumulative distribution function of all storm magnitude statistics is modelled using Copula functions. Comparison of modelled and observed scatterplots shows similar patterns. Goodness-of-fit tests and graphical visualisations show a reasonable agreement between the distribution of modelled synthetic events and observed events. Eventually, a set of long-term synthetic timeseries of storm events $\left(10^{6}\right.$ years $)$ is generated using the event magnitude and timing suggested by the fitted models. These long-term synthetic events can be used to derive exceedance probabilities and to construct designed storm events to be applied to modelling of beach erosion at the site.

\section{ACKNOWLEDGMENTS}

This study is funded by the Bushfire and Natural Hazards CRC Project "Resilience to clustered disaster events on the coast - storm surge". We appreciate the valuable comments from all the reviewers. This paper is published with permission of the Chief Executive Officer, Geoscience Australia.

\section{REFERENCES}

Callaghan, D. P., P. Nielsen, A. Short and R. Ranasinghe (2008). Statistical simulation of wave climate and extreme beach erosion. Coastal Engineering 55(5): 375-390.

Coco, G., N. Senechal, A. Rejas, K. R. Bryan, S. Capo, J. P. Parisot, J. A. Brown and J. H. M. MacMahan (2014). Beach response to a sequence of storms. Geomorphology 204: 493-501.

Corbella, S. and D. D. Stretch (2012). Predicting coastal erosion trends using non-stationary statistics and process-based models. Coastal Engineering 70: 40-49.

De Michele, C., G. Salvadori, G. Passoni and R. Vezzoli (2007). A multivariate model of sea storms using copulas. Coastal Engineering 54(10): 734-751.

Gumbel, E. J. and C. K. Mustafi (1967). Some Analytical Properties of Bivariate Extreme Distributions. $J$. Amer. Stat. Assoc. 62: 569-588.

Hawkes, P. J., B. P. Gouldby, J. A. Tawn and M. W. Owen (2002). The joint probability of waves and water levels in coastal engineering design. Journal of Hydraulic Research 40(3): 241-251.

Hosking, J. R. M. and J. R. Wallis (1988). The effect of intersite dependence on regional flood frequency analysis. Water Resources Research 24(4): 588-600.

Hosking, J. R. M. and J. R. Wallis (1997). Regional Frequency Analysis: An Approach Based on L-Moments. Cambridge Univ. Press, New York.

Karunarathna, H., D. Pender, R. Ranasinghe, A. D. Short and D. E. Reeve (2014). The effects of storm clustering on beach profile variability. Marine Geology 348(2014): 103-112.

Li, F., P. H. A. J. M. van Gelder, R. Ranasinghe, D. P. Callaghan and R. B. Jongejan (2014). Probabilistic modelling of extreme storms along the Dutch coast. Coastal Engineering 86(2014): 1-13.

Lord, D. and M. Kulmar (2000). The 1974 Storms Revisited: 25 Years Experience in Ocean Wave Measurement Along the South-East Australian Coast. Proceedings of the 27th Conference on Coastal Engineering, Sydney, ASCE, 44.

Luceño, A., M. Menéndez and F. J. Méndez (2006). The effect of temporal dependence on the estimation of the frequency of extreme ocean climate events. Proc. R. Soc. A 462: 1683-1697.

Masselink, G. and S. van Heteren (2014). Response of wave-dominated and mixed-energy barriers to storms. Marine Geology 352: 321-347.

Nelsen, R. B. (1999). An Introduction to Copulas. Springer, New York.

R Development Core Team (2012). R: A Language and Environment for Statistical Computing. Vienna: $R$ Foundation for Statistical Computing.

Renard, B. and M. Lang (2007). Use of a Gaussian copula for multivariate extreme value analysis: Some case studies in hydrology. Advances in Water Resources 30(4): 897-912.

Roberts, J., E. C. Leitch, P. G. Lennox and R. Offler (1995). Devonian-Carboniferous stratigraphy of the southern Hastings Block, New England Orogen, eastern Australia. Australian Journal of Earth Sciences 42(6): 609-634.

Royal Haskoning (2013). Old Bar Beach coastal protection structure design investigation. Report to Greater Taree City Council, December 2013. North Sydney, Royal HaskoningDHV: 69.

Schölzel, C. and P. Friederichs (2008). Multivariate non-normally distributed random variables in climate research - introduction to the copula approach. Nonlin. Processes Geophys. 15(5): 761-772.

Serinaldi, F., A. Bárdossy and C. Kilsby (2015). Upper tail dependence in rainfall extremes: would we know it if we saw it? Stochastic Environmental Research and Risk Assessment 29(4): 1211-1233.

Short, A. D. and N. L. Trenaman (1992). Wave Climate of the Sydney Region, an Energetic and Highly Variable Ocean Wave Regime. Australia Journal of Marine and Freshwater Research 43: 26. 